Research Paper

\title{
Influencing Factors of High-Risk Human Papillomavirus Infection and DNA Load According to the Severity of Cervical Lesions in Female Coal Mine Workers of China
}

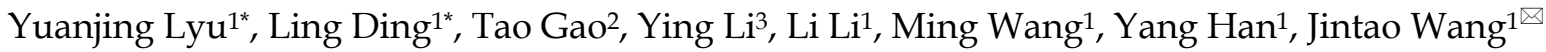 \\ 1. Department of Epidemiology, School of Public Health, Shanxi Medical University, Taiyuan, China. \\ 2. Department of Preventive Medicine, Robert H. Lurie Comprehensive Cancer Center, Feinberg School of Medicine, Northwestern University, Chicago, \\ America. \\ 3. Department of pathology, Jincheng General Hospital, Jincheng, China. \\ * These authors contributed equally to this work. \\ $\triangle$ Corresponding author: Jintao Wang, MD, Ph.D. Department of Epidemiology, School of Public Health, Shanxi Medical University, 56, Xinjian Nan Road, \\ Taiyuan, China. Tel +86 0351-4135245. Email address: wangjt59@163.com. \\ (c) The author(s). This is an open access article distributed under the terms of the Creative Commons Attribution License (https://creativecommons.org/licenses/by/4.0/). \\ See http://ivyspring.com/terms for full terms and conditions.
}

Received: 2019.05.05; Accepted: 2019.07.27; Published: 2019.10.06

\begin{abstract}
High-risk human papillomavirus (HR-HPV) plays an aetiological role in the progression of cervical cancer and precancerous lesions. Determining the risk factors of HR-HPV infection is useful for HR-HPV infection surveillance and control. We aimed to explore the influencing factors of HR-HPV infection in female coal mine workers, and to evaluate the associations between HR-HPV DNA load and cytological and histological changes of cervix. In total 6,325 participants completed standard questionnaire on potential influencing factors of HR-HPV infection and underwent gynecological examinations, HPV test as well as Thinprep cytology test (TCT). 1,512 women with positive results of HPV and/or TCT were referred to colposcopy with biopsy and histological examination. HR-HPV DNA load was evaluated by Digene second generation hybrid capture (HC2) assay. Multiple unconditional logistic regression analysis was used to determine the influencing factors for HR-HPV infection. Of 6,325 study participants, 1,405 (22.2\%) were HR-HPV positive. HR-HPV infection rate was higher in women aged 30-50 years, with lower education level, working inside the mines and engaging in shift work. Risk factors for HR-HPV infection in female coal mine workers included contraception $(\mathrm{OR}=1.395,95 \% \mathrm{Cl}=1.102-1.458)$, previous artificial abortion (OR=1.603, $95 \% \mathrm{Cl}=1.202-1.856)$, working inside the mines $(\mathrm{OR}=1.230,95 \% \mathrm{Cl}=1.056-1.528)$ and history of gynecological diseases $(\mathrm{OR}=1.198,95 \% \mathrm{Cl}=1.001-1.462)$, while menopause was a protective factor $(\mathrm{OR}=0.402,95 \% \mathrm{Cl}=0.306-0.507)$. The HR-HPV DNA load significantly increased with the severity of cervical cytological $\left(X_{\text {trend }}^{2}=177.372, p<0.001\right)$ and histological $\left(X_{\text {trend }}^{2}=194.501, p<0.001\right)$ changes. The results indicated that HR-HPV infection is highly prevalent in female coal mine workers in China. Contraception, artificial abortion, working inside the mines and gynecological diseases could increase the risk of HR-HPV infection in these women. HR-HPV DNA load might predict risks of cervical precancerous lesions and cancer. Our findings could provide scientific basis for reducing the risk of HR-HPV infection and cervical cancer in this vulnerable population.
\end{abstract}

Key words: HR-HPV infection, influencing factors, HR-HPV DNA load, female coal mine workers

\section{Introduction}

Cervical cancer is the fourth most common cancer among females worldwide [1]. In China, there were 98,900 new cases and 30,500 deaths from cervical cancer in 2015, accounting for respectively $18.7 \%$ and $11.5 \%$ of all cervical cancer cases and deaths worldwide [2]. The causal role of high-risk human 
papillomavirus (HR-HPV) in cervical carcinogenesis has been approved by various studies $[3,4]$. HPVs, with an overall prevalence of approximately $10 \%$ (1.4-25.6\%) worldwide, are categorized into HR-HPV and low-risk HPV (LR-HPV) [5]. Geographical differences exist in the prevalence of HPV. According to a pooled analysis, overall HPV prevalence in sub-Saharan Africa was five times higher than in Europe, while HPV prevalence in South America and Asia was intermediate [6]. Several population-based studies in China showed an obvious regional difference of HPV prevalence, and the overall HPV prevalence of Shanxi Province, Shenyang, Shenzhen and Beijing was $14.8 \%, 16.8 \%, 18.4 \%$ and $6.7 \%$, respectively [7-10].

As a coal-rich province in China, Shanxi has a large number of female coal mine workers. Female coal mine workers usually have a relatively lower level of education, younger age of first sexual intercourse, multiple sexual partners, and poor hygiene habits, which have been demonstrated to be risk factors for HPV infection [11,12]. With the humid and poor hygienic conditions in coal mine, it is favorable for micro-organism growth and propagation and harmful for the health of workers $[11,13,14]$. Especially, female coal mine workers could be more prone to HR-HPV infection than the general population. However, epidemiological data on the prevalence and influencing factors of HR-HPV infection in female coal mine workers is scarce. Studies have been done for the association between HPV load and severity of cervical lesions with inconclusive results [15-18]. Thus, in the current study, we aimed to explore the influencing factors of HR-HPV infection in female coal mine workers, and to evaluate the relationships between HR-HPV DNA load and cervical cytological and histological changes as well.

\section{Materials and Methods}

\section{Study population and data collection}

Between September 2011 and October 2012, 6,325 female coal mine workers (age between 20 to 65 years) were enrolled in our study. Subjects were considered eligible for the study according to the following criteria: a) Han people; b) married; c) age $\geq 20$ years old; d) worked in coal mines for at least five years; e) resident in the coal mining area for at least two years; f) not pregnant or lactating; g) no prior history of cervical cancer or precancerous lesions; h) not suffering cancers during the enrollment and has no prior history of tumor of other systems; i) no prior history of treatments for cervix such as Loop Electrosurgical Excision Procedure (LEEP), conization and adnexectomy.

All women enrolled in our study were interviewed by trained interviewers using the structured questionnaire regarding their socio-demographic information, working characteristics, reproductive information, history of gynecological diseases and family history of cancers. Then the subjects underwent a gynecological examination and had samples for HR-HPV detection and Thinprep cytology test (TCT) collected. Colposcopy with biopsy and histological examination were given to women with a positive diagnosis of HR-HPV and/or TCT (Figure 1).

Informed consents have been signed by all the participants, and this study has been approved by the Institutional Review Board of Shanxi Medical University (No. 2008LL07).

\section{HR-HPV Detection}

DNA load of HR-HPV was evaluated by Digene second generation hybrid capture (HC2) DNA test, an in vitro acid hybridization assay with signal amplification using microplate chemiluminescence for HR-HPV DNA detection. A commercial kit detecting HPV types 16, 18, 31, 33, 35, 39, 45, 51, 52, 56, 58,59 and 68 for performing HC2 was used. Alkaline solution was used to denature double-stranded DNA, and the liberated single-strand DNA combined with the RNA probe mix. RNA:DNA hybrids were then

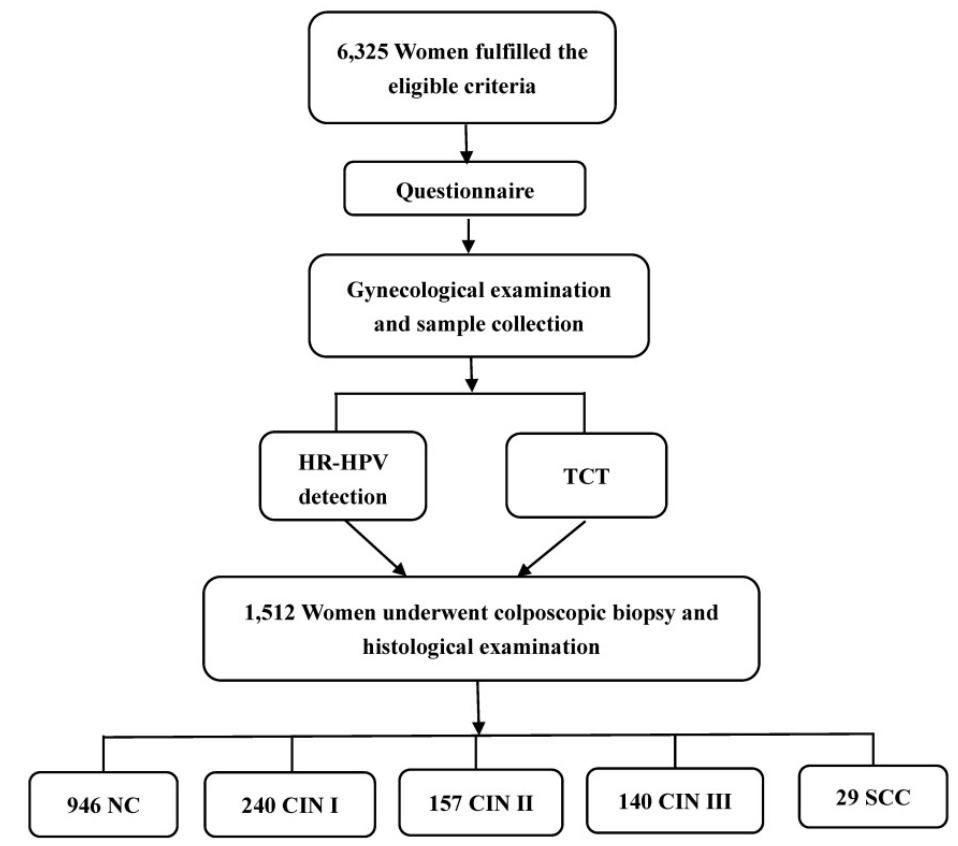

Figure 1. Flowchart of the study population. 
transferred to a capture microplate coated with goat polyclonal anti-RNA:DNA hybrid antibodies to be immobilized. Subsequently, alkaline phosphataseconjugated antibodies to RNA:DNA hybrids were added to react with the bound RNA:DNA hybrids. After washing, chemiluminescent substrate, as the substrate for alkaline phosphatase, was added for signal amplification. Results were analyzed by DIGENE DML 2000 Software. DNA load was expressed by the unit of Relative Light Units/Cutoff Value (RLU/CO), representing the ratio of the light emission of a sample to the average of three positive control samples. HR-HPV DNA load of 1.0 (RLU/CO) or more was defined as HR-HPV positive. HR-HPV viral load was categorized into two groups: low and moderate viral load ( $<100 \mathrm{RLU} / \mathrm{CO})$ and high viral load ( $\geq 100$ RLU/CO) [16].

\section{TCT Test}

Exfoliated cervical cells were collected for cytology test. The cytological evaluation was performed by two cytopathologists at Jincheng General Hospital and the Bethesda Classification system (2001 version) [19] was applied to evaluate cellularity. Diagnosis was classified as follows: a) negative for intraepithelial lesion or malignancy (NILM); b) atypical squamous cells of undetermined significance (ASC-US); c) low-grade squamous intraepithelial lesions (LSIL); d) high-grade squamous intraepithelial lesions (HSIL); e) squamous cell carcinoma (SCC). A diagnosis of ASC-US or worse was considered TCT test positive.

\section{Colposcopy and Cervical Histological Examination}

Colposcopic biopsy was performed in subjects with a positive outcome of HR-HPV and/or TCT. Cervix was divided into quadrants and examined each quadrant by gynecological specialists. All visually abnormal areas were biopsied, and the quadrants without a visible lesion were given random biopsies at the squamo-columnar junction. Histological examinations by biopsy were evaluated together by two pathologists. The cases were classified as normal cervix (NC), cervical intraepithelial neoplasia grade 1 (CIN I), grade 2 (CIN II), grade 3 (CIN III) and squamous cell carcinoma (SCC).

\section{Statistical Analysis}

Influencing factors of HR-HPV infection were analyzed in women with different HR-HPV infection status (HR-HPV-positive and -negative), and associations between HR-HPV DNA load and cervical lesions were analyzed by comparing differences of HR-HPV DNA load in different cervical lesion groups. Count data were examined by Chi-square and trend Chi-square tests. One-way ANOVA was used to analyze differences between groups, and the differences between any two groups were further compared by SNK test. Multivariate unconditional logistic regression was used to determine the influencing factors of HR-HPV infection, and backward stepwise likelihood was used with the inclusion criteria of 0.05 and the exclusion criteria of 0.10 . All the data analyses were performed using Statistical Package for the Social Sciences (SPSS) software version 19.0 (IBM Corporation, Armonk, New York, USA). All reported $p$ values were two-sided, and statistical significance was defined as $p<0.05$.

\section{Results}

\section{Socio-demographic characteristics related to HR-HPV infection}

The mean age of our study subjects was $35.31 \pm 8.89$ years (ranged between 20 and 65 years). Subjects aged between 30 and 50 years old had the highest HPV prevalence (26.5\%), and the prevalence declined as age increased. Only 1,850 women (29.2\%) received higher education (college/university and above). The HR-HPV infection rate significantly decreased with increasing education level $\left(x^{2}\right.$ trend $\left.=35.897, p<0.001\right)$. More than half of the women $(3,572 / 6,325,56.5 \%)$ reported born in Jincheng and the majority $(5,230 / 6,325,82.7 \%)$ were married. However, HR-HPV infection rate showed no significant association with birthplace and marital status $(p>0.05)$. The socio-demographic characteristics of the study subjects are shown in Table 1 .

Table 1. Socio-demographic characteristics related to HR-HPV infection by univariate analysis.

\begin{tabular}{|c|c|c|c|c|c|}
\hline Variable & $\mathrm{n}$ & $\begin{array}{l}\text { HR-HPV } \\
\text { infection } \\
\text { rate }(\%)\end{array}$ & $x^{2}$ & $p$ & OR $(95 \% \mathrm{CI})$ \\
\hline \multicolumn{6}{|l|}{ Age(years) } \\
\hline $20-$ & 857 & 15.1 & & & 1.000 \\
\hline $30-$ & 2156 & 26.3 & 43.662 & $<0.001$ & $2.014(1.632-2.485)$ \\
\hline $40-$ & 2134 & 26.7 & 46.406 & $<0.001$ & $2.057(1.667-2.538)$ \\
\hline $50-$ & 773 & 12.4 & 2.060 & 0.515 & $0.812(0.611-1.079)$ \\
\hline$\geq 60$ & 405 & 10.6 & 4.596 & 0.032 & $0.670(0.464-0.968)$ \\
\hline \multicolumn{6}{|l|}{ Education level } \\
\hline Junior high school and below & 1939 & 25.9 & & & 1.000 \\
\hline Senior high school* & 2536 & 22.7 & 6.064 & 0.014 & $0.841(0.733-0.965)$ \\
\hline College & 768 & 18 & 19.119 & $<0.001$ & $0.627(0.508-0.774)$ \\
\hline University and above & 1082 & 17.5 & 27.922 & $<0.001$ & $0.606(0.503-0.730)$ \\
\hline \multicolumn{6}{|l|}{ Birthplace } \\
\hline Jincheng & 3572 & 22.3 & & & \\
\hline Other cities & 2753 & 22.1 & 0.047 & 0.829 & $1.013(0.899-1.142)$ \\
\hline \multicolumn{6}{|l|}{ Marital status } \\
\hline Married & 5230 & 22.6 & & & \\
\hline Divorced/separated/widowed & 1095 & 20.4 & 2.618 & 0.106 & $1.142(0.972-1.341)$ \\
\hline
\end{tabular}




\section{Influencing factors related to HR-HPV infection}

Of the 6,325 participants, 1,405 (22.2\%) were HR-HPV positive. Univariate analysis showed a significant association between HR-HPV infection and workplace, menopause, contraception, contraceptive method, gravidity, parity, abortion history, artificial abortion history, and the history of gynecological disease (vaginitis, pelvic inflammation disease, uterine fibroids, hyperplasia endometrii, and ovarian tumors) $(p<0.05)$ (Table 2). Multivariate unconditional logistic regression showed that contraception, artificial abortion, working inside the mines and history of gynecological diseases were risk factors for HR-HPV infection, while menopause was a protective factor (Table 3).

Table 2. Potential influencing factors related to HR-HPV infection by univariate analysis.

\begin{tabular}{|c|c|c|c|c|c|}
\hline Variable & $\mathrm{n}$ & $\begin{array}{l}\text { HR-HPV } \\
\text { infection rate (\%) }\end{array}$ & $x^{2}$ & $p$ & OR $(95 \% \mathrm{CI})$ \\
\hline \multicolumn{6}{|l|}{ Workplace } \\
\hline Outside the mines & 3255 & 18.8 & & & 1.000 \\
\hline In the mines & 3070 & 25.9 & 45.966 & $<0.001$ & $1.509(1.339-1.700)$ \\
\hline \multicolumn{6}{|l|}{ Shift work } \\
\hline No & 1587 & 20.9 & & & 1.000 \\
\hline Yes & 4738 & 22.5 & 1.721 & 0.19 & $1.097(0.955-1.261)$ \\
\hline \multicolumn{6}{|l|}{ Menopause } \\
\hline No & 5434 & 21.9 & & & 1.000 \\
\hline Yes & 891 & 15.3 & 20.341 & $<0.001$ & $0.642(0.529-0.780)$ \\
\hline \multicolumn{6}{|l|}{ Contraception } \\
\hline No & 1860 & 19.6 & & & 1.000 \\
\hline Yes & 4465 & 23.3 & 10.227 & $<0.001$ & $1.244(1.088-1.422)$ \\
\hline \multicolumn{6}{|c|}{ Contraceptive method } \\
\hline Condom & 601 & 13.5 & & & 1.000 \\
\hline IUD & 2284 & 51.2 & 275.472 & $<0.001$ & $5.731(5.252-8.626)$ \\
\hline OC & 1580 & 35.4 & 100.735 & $<0.001$ & $3.515(2.721-4.540)$ \\
\hline \multicolumn{6}{|l|}{ Gravidity } \\
\hline$\leq 1$ & 2577 & 20.3 & & & 1.000 \\
\hline $2-3$ & 3349 & 21.9 & 2.210 & 0.137 & $1.100(0.970-1.248)$ \\
\hline$>3$ & 399 & 37.1 & 61.362 & $<0.001$ & $2.422(1.932-3.037)$ \\
\hline \multicolumn{6}{|l|}{ Parity } \\
\hline$\leq 1$ & 5520 & 21.7 & & & 1.000 \\
\hline$\geq 2$ & 805 & 25.8 & 6.950 & 0.008 & $1.257(1.060-1.490)$ \\
\hline \multicolumn{6}{|l|}{ Abortion history } \\
\hline No & 2993 & 19.2 & & & 1.000 \\
\hline Yes & 3332 & 24.9 & 29.631 & $<0.001$ & $1.395(1.237-1.573)$ \\
\hline \multicolumn{6}{|l|}{ Artificial abortion } \\
\hline No & 3371 & 23.1 & & & 1.000 \\
\hline Yes & 2954 & 25.5 & 37.272 & $<0.001$ & 1.439(1.28.-1.618) \\
\hline \multicolumn{6}{|c|}{$\begin{array}{l}\text { History of } \\
\text { gynecological diseases }\end{array}$} \\
\hline No & 5897 & 21.6 & & & 1.000 \\
\hline Yes & 428 & 30.4 & 17.770 & $<0.001$ & $1.583(1.277-1.963)$ \\
\hline
\end{tabular}

\section{HR-HPV DNA load according to cervical cytological and histological changes}

In all 6,325 subjects, colposcopy with biopsy and histological examination were given to 1,512 women with a positive diagnosis of HR-HPV and/or TCT. Among them, 946 were diagnosed with NC, 240 with
CIN I, 157 with CIN II, 140 with CIN III and 29 with SCC. Associations between HR-HPV DNA load and cytological and histological changes are shown in Table 4 and Figure 2. HR-HPV DNA load increased with the severity of cervical cytological and histological changes $(p<0.05)$. High viral load of HR-HPV was found to be significantly related to cytological $\left(\chi^{2}\right.$ trend $\left.=177.372, p<0.001\right)$ and histological $\left(\chi^{2}{ }_{\text {trend }}=194.501, p<0.001\right)$ changes of cervix.

Table 3. Influencing factors for HR-HPV infection assessed by multivariate unconditional logistic regression.

\begin{tabular}{|c|c|c|c|c|}
\hline Variables & $\beta$ & $S_{b}$ & Wald $\chi^{2}$ & $\mathrm{OR}^{*}(95 \% \mathrm{CI})$ \\
\hline Menopause & -0.629 & 0.112 & 73.102 & $0.402(0.306-0.507)$ \\
\hline Contraception & 0.196 & 0.079 & 4.961 & $1.395(1.102-1.458)$ \\
\hline Artificial abortion & 0.512 & 0.152 & 9.362 & $1.603(1.202-1.856)$ \\
\hline Working inside the mines & 0.183 & 0.105 & 6.587 & $1.230(1.056-1.528)$ \\
\hline History of gynecological diseases & 0.175 & 0.120 & 2.018 & $1.198(1.001-1.462)$ \\
\hline
\end{tabular}

\section{Discussion}

In this study, we found that female coal mine workers had a relatively higher prevalence of HR-HPV with 22.2\%, which was higher than population-based prevalence observed in Shanxi Province (12.2\%), Shenyang (11.7\%), Shenzhen $(13.5 \%)$ and Beijing $(5.8 \%)$, suggesting that female coal mine workers might have a higher risk for cervical cancer [7-10].

In our study, HR-HPV prevalence in female coal mine workers reached peak at ages 30-50, which might be attributable to the relatively active sex life in women of this age as several studies have shown that HPV could be transmitted to cervix efficiently by vaginal intercourse [20]. We also found that HR-HPV infection rate decreased with increasing education level, which is in consistent with previous studies [21]. Shift work could result in a series of negative effects on health, such as adverse effects on hormone and reproductive function of females [22]. In this study, the HR-HPV infection rate in shift workers was higher than non-shift workers, though it didn't reach a statistical level. Improving the working environment of the coal mines and developing cervical cancer screening strategies for coal miners may be particularly efficient. In addition, women working inside the mines have 1.230 higher risk of HR-HPV infection than workers working outside the mines, which may be due to the fact that the humid and poor hygienic conditions in the mines can promote the growth of microbe $[11,13,14]$.

In our study, significant increase in the risk of HPV infection was observed in women using contraceptives, who have 1.395 higher risk of HR-HPV infection than women who did not, which is 


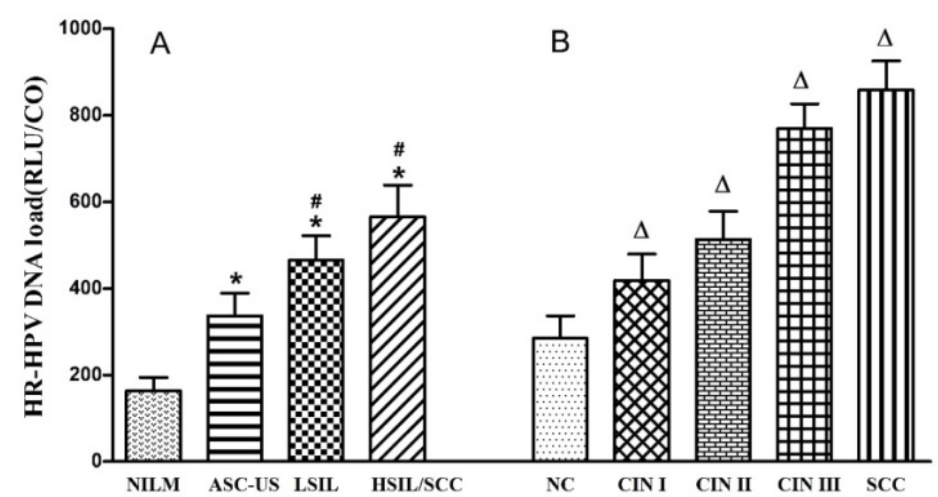

Figure 2. HR-HPV DNA load among different cytological (A) and histological (B) changes. HR-HPV load increased with severity of cervical cytological and histological changes. *, Difference showed a statistical significance when compared to NILM $(p<0.05)$; \#, difference showed a statistical significance when compared to ASC-US ( $p<0.05)$; $\Delta$, difference showed a statistical significance when compared to NC $(p<0.05)$.

similar to a former study [23]. Majority (86.5\%) of the subjects in our study chose intrauterine device (IUD) and oral contraceptives (OC) as contraceptive methods. Abnormal bleeding induced by IUD could increase the risk for HPV infection. Moreover, OC has been shown to enhance hydroxylation of estradiol to 16a-hydroxyestrone in cervical cells infected with HR-HPV, which induces increased transcription of the HPV oncogenes E6 and E7 [24]. In addition, menopause was found to be a protective factor of HR-HPV infection in our study, and the HR-HPV risk in postmenopausal women was only half of that in premenopausal women. Our previous studies have shown that menopause was a protective factor, which could be ascribed to the decreased estrogen level of menopause women $[25,26]$. Therefore, we suggest the use of condoms instead of OC or IUD for contraception to effectively reduce HPV infection.

Our results showed that artificial abortion was also a risk factor for HR-HPV infection. Artificial abortion can cause damage to the endometrium and mechanical injury to the cervix, so as result in
HR-HPV infection. In this study, history of gynecological diseases was also a risk factor for HR-HPV infection. Several studies reported that women with gynecological diseases were prone to persistent HR-HPV infection due to unstable sexual hormone levels, poor function of immune system, lower resistance to HR-HPV and weak ability of viral clearance $[27,28]$. Our results suggested that active treatment for gynecological diseases is of great importance to reduce the risk for HR-HPV infection.

Results of this study also showed that HR-HPV DNA load and the proportion of high viral load increased with the severity of cervical cytological and histological changes. Huang et al [29] reported that the risk of cervical precancerous lesions and cervical cancer significantly increased when HPV DNA load was higher than $100 \mathrm{RLU} / \mathrm{CO}$ and $500 \mathrm{RLU} / \mathrm{CO}$ respectively, which was consistent with our results. Dalstein $\mathrm{V}$ et al [30] reported that high viral load could be used as a short-term marker of progression towards CIN from a prospective study. Moreover, another cohort study revealed that the progression to CIN II/III was linked to HPV 16 DNA load which evaluated by quantitative real-time PCR, and the dynamic change of HPV viral load was associated with cervical lesions [31]. Peitsaro et al [32] reported that high viral load was likely to increase the risk for viral integration with the host, and this could partly explain the relationship between viral load and cervical lesions. Furthermore, the possible use of the HPV load to predict the degree of cervical lesion has been proposed $[33,34]$. It is beneficial to carry out antivirus therapy in women with high HR-HPV load to reduce the risk for cervical cancer in this population.

Table 4. Relationships between HR-HPV DNA load and cervical lesions

\begin{tabular}{|c|c|c|c|c|c|c|}
\hline \multirow[t]{2}{*}{ Cervical lesions } & \multirow[t]{2}{*}{$\overline{\mathrm{x}} \pm \mathrm{s}$} & \multicolumn{2}{|c|}{ HR-HPV DNA load } & \multirow[t]{2}{*}{$x^{2}$} & \multirow[t]{2}{*}{$p$} & \multirow[t]{2}{*}{ OR $(95 \% \mathrm{CI})$} \\
\hline & & $\mathrm{L}(\%)$ & $\mathrm{H}(\%)$ & & & \\
\hline \multicolumn{7}{|c|}{ Cytological change } \\
\hline NILM & $163.850 \pm 30.170$ & $4505(77.5 \%)$ & $1308(22.5 \%)$ & & & 1.000 \\
\hline ASC-US & $336.780 \pm 51.961^{*}$ & $169(58.0 \%)$ & $122(42.0 \%)$ & 58.280 & $<0.001$ & $2.486(1.954-3.163)$ \\
\hline LSIL & $465.170 \pm 56.192^{*} \#$ & $63(45.4 \%)$ & $76(54.6 \%)$ & 78.750 & $<0.001$ & $4.155(2.959-5.835)$ \\
\hline \multirow[t]{2}{*}{$\mathrm{HSIL} / \mathrm{SCC}$} & $565.230 \pm 73.557^{*} \#$ & $33(40.4 \%)$ & $49(59.6 \%)$ & 63.331 & $<0.001$ & $5.114(3.275-7.986)$ \\
\hline & $\mathrm{F}=44.848, p<0.001$ & \multicolumn{5}{|c|}{$\chi^{2}=183.394, p<0.001 ; \chi^{2}$ trend $=177.372, p<0.001$} \\
\hline \multicolumn{7}{|c|}{ Histological change } \\
\hline NC & $284.60 \pm 52.108$ & $618(65.3 \%)$ & $328(34.7 \%)$ & & & 1.000 \\
\hline CIN I & $417.88 \pm 61.102 \Delta$ & $109(45.4 \%)$ & $131(54.6 \%)$ & 31.991 & $<0.001$ & $2.264(1.699-3.018)$ \\
\hline CIN II & $513.12 \pm 64.587 \Delta$ & $57(36.3 \%)$ & $100(63.7 \%)$ & 47.761 & $<0.001$ & $3.306(2.325-4.699)$ \\
\hline CIN III & $769.51 \pm 56.680 \Delta$ & $16(11.4 \%)$ & $124(88.6 \%)$ & 145.809 & $<0.001$ & $14.809(8.530-24.997)$ \\
\hline \multirow[t]{2}{*}{ SCC } & $859.32 \pm 65.596 \Delta$ & $3(10.3 \%)$ & $26(89.7 \%)$ & 36.784 & $<0.001$ & $22.481(4.906-54.354)$ \\
\hline & $\mathrm{F}=12.440, p<0.001$ & \multicolumn{5}{|c|}{$\chi^{2}=199.176, p<0.001 ; \chi^{2}$ trend $=194.501, p<0.001$} \\
\hline
\end{tabular}

OR, odds ratio; $\mathrm{CI}$, confidence interval; ${ }^{*}$, Difference showed a statistical significance when compared to NILM ( $\left.p<0.05\right)$; \#, difference showed a statistical significance when compared to ASC-US; $\Delta$, difference showed a statistical significance when compared to NC; L, low and moderate HR-HPV DNA load; H, high HR-HPV DNA load. 
In conclusion, in this study we assessed the influencing factors of HR-HPV infection in female coal mine workers, and found that contraception, artificial abortion, working inside the mines and gynecological diseases could increase the risk of HR-HPV infection. HR-HPV DNA load could predict the risk of cervical precancerous lesions and cancer based on this cross-sectional study. Our findings may provide scientific basis to reduce the risk of HR-HPV infection and cervical cancer in this vulnerable population. Certainly, out of the limitations of the research method we used, the ability to prove the hypothesis of etiology was relatively weak. The prospective cohort studies will be needed to provide more powerful evidence.

\section{Acknowledgments}

We gratefully acknowledge the contribution of our coworkers involved in conducting the study and writing this article. We thank all the study participants, as well as the investigators and local healthcare workers from Jincheng General Hospital for their help with this study.

This study was supported by the National Natural Science Foundation of China (No: 81473060, 81273157, and 30872166) and Special Public Welfare Industry Research of National Health and Family Planning Commission, China (No: 201402010).

\section{Competing Interests}

The authors have declared that no competing interest exists.

\section{References}

1. Bray F, Ferlay J, Soerjomataram I, et al. Global Cancer Statistics 2018: GLOBOCAN Estimates of Incidence and Mortality Worldwide for 36 Cancers in 185 Countries[J]. CA Cancer J Clin. 2018;68(6):605-8.

2. Chen $\mathrm{W}$, Zheng R, Baade PD, et al. Cancer statistics in China, 2015. CA Cancer J Clin. 2016; 66(2):115-32.

3. Ginsburg O, Bray F, Coleman MP, et al. The global burden of women's cancers: a grand challenge in global health. Lancet. 2016; 389(10071):847-60.

4. Senapati R, Nayak B, Kar SK, et al. HPV Genotypes distribution in Indian women with and without cervical carcinoma: Implication for HPV vaccination program in Odisha, Eastern India. BMC Infectious Diseases. 2017; 17(1):30.

5. Guo T, Gao Y, Gao Z, et al. Human papillomavirus genotype distribution among HPV-positive women in Sichuan province, Southwest China. Arch Virol. 2018; 163(1):65.

6. Clifford GM, Gallus S, Herrero R, et al. Worldwide distribution of human papillomavirus types in cytologically normal women in the International Agency for Research on Cancer HPV prevalence surveys: a pooled analysis. Lancet. 2005; 366(9490):991-8.

7. Dai M, Bao YP, Li N, et al. Human papillomavirus infection in Shanxi Province, People's Republic of China: a population-based study. Br J Cancer. 2006; 95(1):96-101.

8. Li LK, Dai M, Clifford G M, et al. Human papillomavirus infection in Shenyang City, People's Republic of China: a population-based study. Br J Cancer. 2006; 95(11):1593-7.

9. Wu RF, Dai M, Qiao YL, et al. Human papillomavirus infection in women in Shenzhen City, People's Republic of China, a population typical of recent Chinese urbanization. Int J Cancer. 2007; 121(6):1306-11.

10. Zhao R, Zhang WY, Wu MH, et al. Human papillomavirus infection in Beijing, People's Republic of China: a population-based study. Br J Cancer. 2009; 101(9):1635-40

11. Liu L, Wang L, Chen J. Prevalence and associated factors of depressive symptoms among Chinese underground coal miners. Biomed Research International. 2014; 2014(2): 987305
12. Ribeiro AA, Costa $\mathrm{MC}$, Alves RR, et al. HPV infection and cervical neoplasia: associated risk factors. Infectious Agents and Cancer. 2015; 10:16.

13. Considine R, Tynan R, James C, et al. The Contribution of Individual, Social and Work Characteristics to Employee Mental Health in a Coal Mining Industry Population. PLoS ONE. 2017; 12(1): e0168445.

14. Liu H, Tang Z, Yang $\mathrm{Y}$, et al. Identification and classification of high risk groups for Coal Workers' Pneumoconiosis using an artificial neural network based on occupational histories: a retrospective cohort study. BMC Public Health. 2009; 9:366

15. Moberg M, Gustavsson I, Wilander E, et al. High viral loads of human papillomavirus predict risk of invasive cervical carcinoma. Br J Cancer. 2005; 92(5):891-4.

16. Xu Y, Dotto J, Hui $Y$, et al. High grade cervical intraepithelial neoplasia and viral load of high-risk human papillomavirus: significant correlations in patients of 22 years old or younger. Int Journal Clin Exp Pathol. 2009; 2(2):169-75.

17. Andersson S, Safari H, Mints $\mathrm{M}$, et al. Type distribution, viral load and integration status of high-risk human papillomaviruses in pre-stages of cervical cancer (CIN). Br J Cancer. 2005; 92(12):2195-200

18. Manawapat-Klopfer A, Wang L, Haedicke-Jarboui J, et al. HPV16 viral load and physical state measurement as a potential immediate triage strategy for HR-HPV-infected women: a study in 644 women with single HPV16 infections. Am J Cancer Res. 2018; 8(4): 715-22.

19. Solomon D, Davey D, Kurman R, et al. The 2001 Bethesda System: terminology for reporting results of cervical cytology. JAMA. 2002; 287:2114-9.

20. Widdice LE, Moscicki AB. Updated guidelines for papanicolaou tests, colposcopy, and human papillomavirus testing in adolescents. Kowalski R M, Limber S P. Electronic bullying among middle school students. I Adolesc Health: Official Publication of the Society for Adolescent Medicine. 2008; 43(4 Suppl): S41-51.

21. Wu MH, Zhang SW, Zhang WY, et al. Epidemiological study of high risk human papillomavirus infection in 25 to 54 years old married women in Beijing. Zhonghua Fu Chan Ke Za Zhi. 2009; 44(12):892-7.

22. Harrington JM. Health effects of shift work and extended hours of work. Occup Environ Med. 2001; 58(1):68-72.

23. Vinodhini K, Shanmughapriya S, Das BC, et al. Prevalence and risk factors of HPV infection among women from various provinces of the world. Arch Gynecol Obstet. 2012; 285(3):771-7.

24. de Villiers EM. Relationship between steroid hormone contraceptives and $\mathrm{HPV}$, cervical intraepithelial neoplasia and cervical carcinoma. Int J Cancer. 2003; 103(6):705-8

25. Wang JT, Gao ES, Cheng YY, et al. Analysis on synergistic action between estrogen, progesterone and human papillomaviruses in cervical cancer. Chinese Journal of Epidemiology. 2005; 26(5):370-3.

26. Wang JT, Gao ES, Ding L, et al. Association between endogenous hormones, hormone receptors and cervical cancer. Chinese journal of oncology. 2006; 28(7):494-7.

27. Bahmanyar ER, Paavonen $J$, Naud $P$, et al Prevalence and risk factors for cervical HPV infection and abnormalities in young adult women at enrolment in the multinational PATRICIA trial. Gynecologic Oncology. 2012; 127(3):440-50

28. Li Y, Xiang Y, Zhang RF, et al. Viral load, genomic integration frequency of human papillomavirus 16 in cervical cancer and precancerous lesions. National Medical Journal of China. 2011; 91(13): 906-10.

29. Huang ZH, Oian DY, Wang D, et al. Study of Relationship Between Loads of Human Papilloma Virus in Cervical Carcinoma and Cervical Intraepithelial Neoplasia. Maternal and Child Health Care of China. 2006,21(11):1557-9.

30. Dalstein V, Riethmuller D, Prétet JL, et al. Persistence and load of high-risk HPV are predictors for development of high-grade cervical lesions: A longitudinal French cohort study. Int J Cancer. 2003; 106(3):396-403.

31. Monnier-Benoit S, Dalstein V, Riethmuller D, et al. Dynamics of HPV16 DNA load reflect the natural history of cervical HPV-associated lesions. Journal of Clinical Virology. 2006; 35(3):270-7.

32. Peitsaro $P$, Johansson $B$, Syrjänen S. Integrated human papillomavirus type 16 is frequently found in cervical cancer precursors as demonstrated by a novel quantitative real-time PCR technique. Journal of Clinical Microbiology. 2002, 40(3):886-91.

33. Schlecht NF, Trevisan A, Duarte-Franco E, et al. Viral load as a predictor of the risk of cervical intraepithelial neoplasia. Int J Cancer. 2003; 103(4):519-24.

34. Fiander AN, Hart KW, Hibbitts SJ, et al. Variation in human papillomavirus type-16 viral load within different histological grades of cervical neoplasia. Journal of Medical Virology. 2007; 79(9):1366-9. 